\title{
Energy Efficient Clustering Protocol for Wireless Sensor Network
}

\author{
Shraddha Agrawal ${ }^{\# 1}$, Rajeev Pandey ${ }^{\# 2}$, Mahesh Motwani ${ }^{\# 3}$

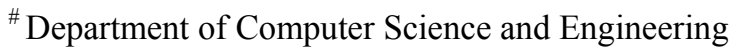 \\ UIT RGPV, Bhopal, India \\ 145shraddha@gmail.com
}

\begin{abstract}
Energy consumption of sensor nodes in wireless sensor networks (WSNs) is a big challenge for improving network lifetime. Several approaches have been proposed in existing literature for this. One of the best approach can be considered as clustering. During clustering strategy, cluster head (CH) selection plays an important role because $\mathrm{CH}$ is responsible for collecting data from the member nodes, processing and forwarding the data to next level or sink. Because of this, energy requirement for $\mathrm{CH}$ is more than member nodes. In our work we have tried to minimize energy consumption of a network by selecting optimal $\mathrm{CH}$ in WSNs using energy and distance parameters. In this paper we propose $\mathrm{CH}$ selection algorithm using energy and distance (E/D) parameter based on fan-shaped design. Experimental results prove that our method has advantages of selecting $\mathrm{CH}$ over random $\mathrm{CH}$ selection method.
\end{abstract}

Keyword- Wireless Sensor Network, Clustering, fan-shaped clustering

\section{INTRODUCTION}

Wireless sensor network consists of various small, limited energy nodes distributed across the field. These nodes are responsible for sensing the environmental data and forwarding to the destination or sink. Mostly these nodes are deployed in remote location where energy sources or supply is limited which imposes a restriction to recharge the batteries of these nodes. Hence the communication among the nodes should consume minimum power to increase network lifetime. Various methods or approaches have been proposed in existing literature for this [4], [5], [6], [7]. One of the techniques is effective clustering [2] [3].

Clustering divides the network into smaller partitions. Each partition is known as cluster. Each cluster selects a $\mathrm{CH}$ which is responsible for collecting data from member nodes and forwarding it to the next cluster or sink during clustering process.

$\mathrm{CH}$ is a node which consumes more power than member nodes. Hence it should be selected optimally to improve the network performance. Cluster based technique in wireless sensor network employees two types of communication namely intra-cluster communication and inter-cluster communication. In intra-cluster communication the member nodes of a cluster selects a $\mathrm{CH}$ and send data to it. During inter-cluster communication one $\mathrm{CH}$ will send the aggregated data of a cluster to another $\mathrm{CH}$.

In our work we have focused on effective communication strategy to select best $\mathrm{CH}$ so that energy spent in communication should be minimized resulting into optimal network utilization. Our work is based on the fanshaped clustering model proposed by Hai Lin et.al [1]. The whole network is divided into fan-shaped clusters in which sink occupies the center position.

In this paper we optimize the $\mathrm{CH}$ selection procedure by considering following two parameters based on fan shaped architecture.

1. Energy of a node and

2. Distance of a node from sink

The remainder of this paper is organized as follows: Section 2 describes the clustering technique and its related works. Section 3 describes our E/D approach in detail specifically the CH selection formula. Section 4 describes Random cluster head selection approach and Section 5 analyses the performance of our technique through experiments. Section 6 summarizes the work with the conclusion.

\section{RELATED WORK}

Earlier work in clustering such as LEACH [5], focused on random selection of CH. LEACH uses single hop routing between $\mathrm{CH}$ and sink. Hence it is not suitable for large scale network. It doesn't guarantee the equal distribution of nodes in clusters. It also ignores the relevant information of neighboring clusters [11]. In EECS [6] $\mathrm{CH}$ selection process consider the residual energy of all eligible nodes. It also focuses on single hop transmission from $\mathrm{CH}$ to Base station like LEACH protocol. In [8] author's select $\mathrm{CH}$ based on energy and 
delay, called as EDIT. SEECH is a distributed protocol proposed by [10] where $\mathrm{CH}$ selection and relay node selection is separated to save network energy. In HEED [7] main focus is on minimizing intra-cluster communication cost. This is achieved by average minimum reachability power measurement (AMRP).

\section{A.Clustering strategy}

Clustering can be performed using different criteria. For example, network is first partitioned into clusters and then $\mathrm{CH}$ is selected for each cluster [14] [15]. Another way of clustering is select the $\mathrm{CH}$ first and then remaining nodes will join $\mathrm{CH}$ to form a cluster [4] [9] [16].

Clustering can also be performed based upon location of nodes. But this type of method requires that every node must be aware of its location. This type of clustering is known as location based clustering [14] [15]. Contrary to this approach clustering can be performed without knowing the location of nodes. This approach is known as non-location based clustering [4] [7].

Furthermore clustering can be classified as one hop or multi hop clustering. In single hop routing every node can directly communicate with each other or the cluster head [4] [7]. In large scale WSN nodes have to pass several hops to reach $\mathrm{CH}$ is known as multi hop clustering [15].

In addition to the above mentioned techniques few other popular techniques are centric and distributed clustering. In centric clustering, there exists a centralized authority like base station which is responsible for selecting the $\mathrm{CH}$ [12] whereas in distributed clustering approach every $\mathrm{CH}$ is responsible for generating its own cluster using distributed methods [4] [12].

\section{B. Cluster head selection}

Cluster head is a very special node which collects the data from its member nodes and passes it to the next level or sink. Clearly, it consumes more energy than its member nodes. In LEACH [5] the cluster head is selected dynamically based on how often and the last time a given node has been elected as a $\mathrm{CH}$ in the past. In EECS [6] only the residual energy is considered for cluster head selection. Where as in HEED [7] residual energy and average minimum reachability power is used for cluster head selection. EDIT [8] considers energy and delay both for the $\mathrm{CH}$ selection.

The $\mathrm{CH}$ selection process in fan-shaped clustering method described in [1] is that every node whose residual energy is greater than threshold sets a random back off time. The first node whose back off time elapses broadcast a winning message that it should be selected as a $\mathrm{CH}$ and all the other nodes cancel their back off time.

We believe that distance should also be considered in combination with residual energy of a node for effective cluster head selection. Because more will be the distance of source from sink, more transmission power it will require to send the data.

\section{Relay selection}

In large scale WSN the collected data can be sent to the sink via some intermediate nodes which are known as relay nodes. In traditional algorithms [5] [7] the same node performs both the task of $\mathrm{CH}$ as well as relay node. If a same node perform both the task than energy will deplete more quickly. To avoid this problem, SEECH [10] has separated both the roles of $\mathrm{CH}$ and relay node. In our work we have also divided the task of the $\mathrm{CH}$ and relay node.

\section{Routing}

To find the optimal route between the source and the sink for data transmission is known as routing. As per LIN et al. [1] in fan shaped clustering packets needs to be forwarded only to the next inner layer.

The main contributions of our paper is, based on fan-shaped clustering method mentioned in [1] we introduce a new $\mathrm{CH}$ and relay node selection method which is based on residual energy of a node and distance in the context. In the next section we would present our work in detail.

An easy way to comply with the conference paper formatting requirements is to use this document as a template and simply type your text into it. 


\section{ENERGY AND DISTANCE (E/D) BASED CLUSTERING APPROACH}

Our clustering protocol which uses energy and distance (E/D) parameters for $\mathrm{CH}$ selection is developed by modifying the model proposed by Hai Lin et.al [1]. Following are the assumptions for our work.

- Sensor nodes are randomly and independently deployed in sensor field.

- All the nodes have the same transmission power and packet length is also fixed.

- Every sensor node is aware of its polar coordinate to the sink node.

- In our work we have given three roles to a sensor node, i.e. cluster head, member node, and relay node.

The network is divided into concentric circular rings (layers). All these layers are at equal distance from each other denoted as $r$. Each layer is then divided into clusters using formula $(2 i-1)^{*} n$, where $i$ indicates the layer number and $n=4$, for square shaped cluster [1]. Cluster size is an important parameter which impacts the network performance. Cluster size should be chosen considering the transmission range of a node. While defining cluster size it should be carefully noted that transmission range of nodes should span across not only its own cluster but neighboring cluster also. For this node's transmission radius (R) should cover maximum distance between the two adjacent clusters. Hence width of layer $r$ is obtained by LIN et al. in [1] as below.

$$
r=R / 3.7318
$$

\section{A. Cluster Head Selection}

If all the nodes in a sensor network, directly sends data to the sink than it will be very difficult for sink to collect and process tremendous amount of data. To avoid the bottleneck the concept of cluster head pitched in. Here, all the member nodes of a cluster elect one cluster head among them. In existing literature, we can find various techniques for selecting cluster head [4], [7], [8], [9], [10].

$\mathrm{CH}$ is an important node, because it collects data from the member nodes, aggregate and forwards to nodes are involved in intra-cluster communication only. But $\mathrm{CH}$ is involved in intra as well as inter-cluster communication. The collected data from the sensor node is ultimately transmitted to the sink via $\mathrm{CH}$. So the distance of $\mathrm{CH}$ from the sink should be minimized for inter-cluster communication.

We can consider that the energy consumed by a $\mathrm{CH}$ depends upon the distance from $\mathrm{CH}$ to the destination. So $\mathrm{CH}$ should be selected depending upon the remaining energy of a node (residual energy) and a distance from destination. Energy (E) consumed by $\mathrm{CH}$ for sending data is directly proportional to the distance (D) of a $\mathrm{CH}$ from the destination.

$$
\begin{aligned}
& \mathrm{E} \alpha \mathrm{D} \\
& \mathrm{E}=\mathrm{k} \mathrm{D} \\
& \mathrm{k}=\mathrm{E} / \mathrm{D}
\end{aligned}
$$

Where $\mathrm{k}$ is a score to decide $\mathrm{CH}$.

The procedure of cluster head selection is the following:

Assuming sink is located at the center and fan shaped clustering has been applied. Let source node(s) wants to send data to sink (d) then -

1) Identify the layer number and cluster number this source belongs to.

2) Within this cluster all the nodes whose residual energy is greater than threshold will further participate in a process for cluster head selection.

3) For all the eligible nodes then score will be calculated using equation (2)

4) The node with maximum score is selected as a cluster head. Here score provides the optimal value based on the residual energy and distance of a cluster head from the sink.

5) After completion of every round score will be calculated for cluster head selection.

6) If energy of current cluster head goes below the threshold then this node will not participate further. 


\section{B. Relay node selection and routing}

Usually the source is far away from sink then it is difficult for a cluster head to communicate in one hop. Hence the data is transferred using intermediate nodes between source cluster head and sink. This intermediate node which helps in effective data transmission is known as relay nodes. The main purpose of a relay node is during routing. Sending data from source to sink is known as routing. During routing source will send data to its cluster head. Next, cluster head will search for relay node in next lower layer. Here we will again apply score formula described in equation (2) to select relay node. For the first time the distance will be considered from the cluster head of source cluster. For further transmission and relay node selection distance will be considered from upper layer relay node. Ultimately the data will be transmitted to the sink.

\section{IV.RANDOM CLUSTER HEAD SELECTION APPROACH}

To better analyze our energy and distance (E/D) based algorithm we have created a parallel approach which selects $\mathrm{CH}$ in random fashion. This approach is developed by modifying the model proposed by Hai Lin et.al in [1]. In this method we have kept all the initial parameters and assumptions same as in E/D method. The only difference is, instead of selecting $\mathrm{CH}$ using E/D formula, it selects $\mathrm{CH}$ randomly.

\section{SIMULATION PARAMETERS AND EXPERIMENTAL RESULTS}

TABLE I. Simulation Parameters

\begin{tabular}{|c|c|}
\hline Parameters & Values \\
\hline Node number & 20 \\
\hline Node initial energy & $300 \mathrm{~mJ}$ \\
\hline Packet length & $2000 \mathrm{bit}$ \\
\hline Energy threshold for $\mathrm{CH}$ and relay & $50 \mathrm{~mJ}$ \\
\hline Node's transmission radius & $300 \mathrm{~m}$ \\
\hline
\end{tabular}

In proposed mechanism we have used NS2.34 as simulation platform. Nodes are randomly deployed and then partitioned into circular rings in such a manner that sink is located at the center. Every node will send one packet in one round (one second). All the initial parameter settings are listed in Table I.

\section{A. Performance analysis}

The performance is evaluated based on three criterions. 1. Number of alive nodes, 2. Total residual energy and 3. Packet collection rate.

1) Number of alive nodes: After each round we will check the residual energy of all the nodes. Nodes whose residual energy is above a predefined threshold is considered as alive nodes.

Our task is to improve number of alive nodes after each round.

2) Residual energy: It can be termed as sum of energies of all the alive nodes in network. So, our goal is to minimize the energy consumption.

3) Packet collection rate (PCR): PCR for each round is defined as ratio of number of actual packet received by the sink and total number of alive nodes.

In the following each simulation is executed 8 to 10 times and then mean value is used for performance analysis. All the three parameters are analyzed with respect to time assuming one round is for one second. From the Fig. 1 we can notice that no node dies until 230 seconds than nodes start dying gradually up to round 245. And then from 245 seconds onwards we have noticed that all the nodes are dead. By this behavior we can conclude that our approach of selecting $\mathrm{CH}$ based on energy and distance (E/D) formula distributed the task of $\mathrm{CH}$ evenly among the eligible nodes and allows graceful degradation till round 245 providing good load balancing. Same Thing can be observed in Fig. 2 where residual energy of a network decreases proportionally with time. We can observe in Fig. 3 that packet collection rate is almost $100 \%$ with few drops. 


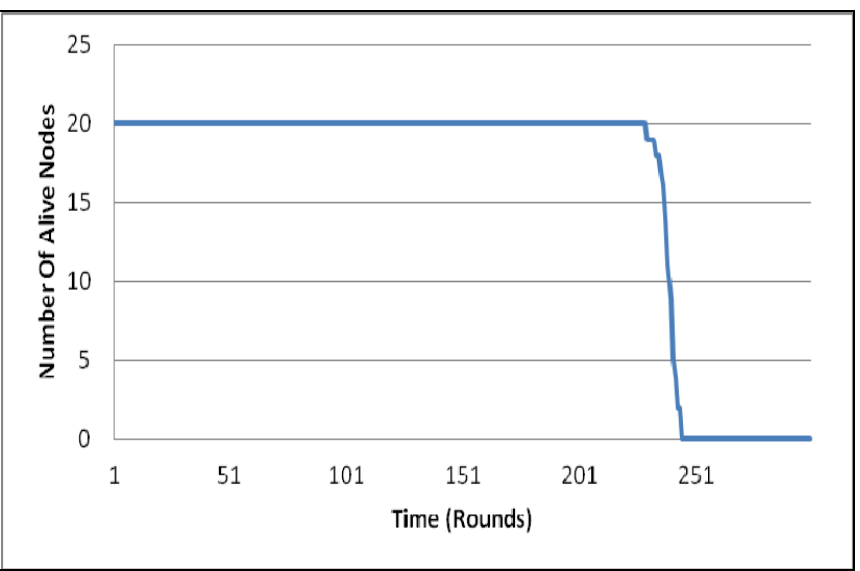

Fig. 1. Number of Alive nodes Vs. Time

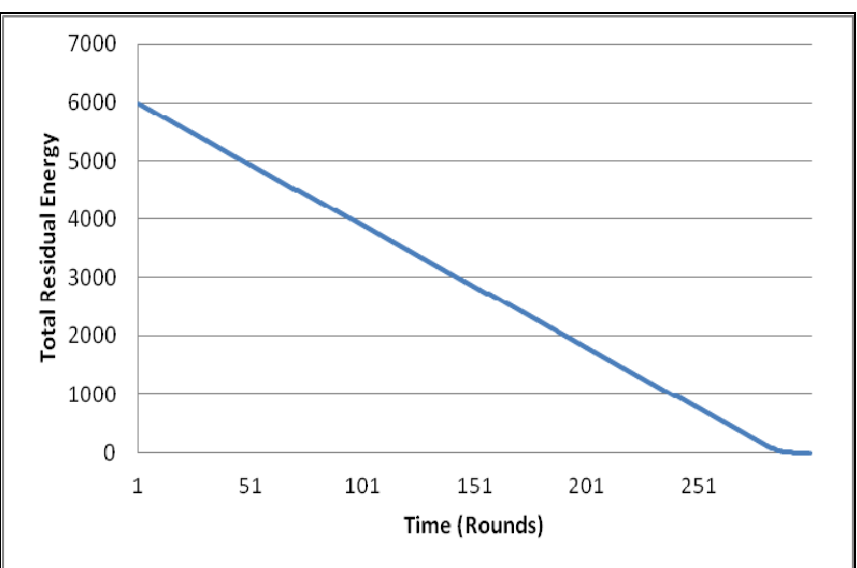

Fig. 2. Total Residual Energy Vs. Time

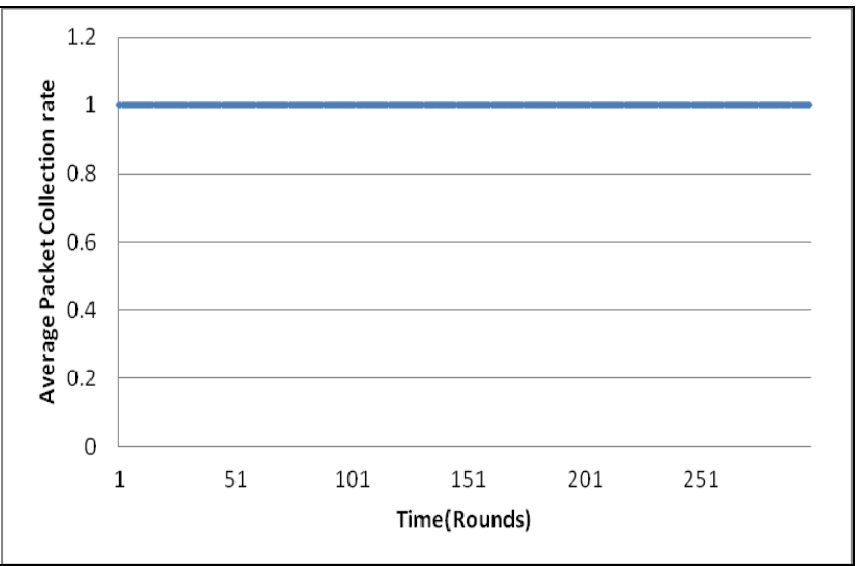

Fig. 3. Average Packet Collection Rate Vs. Time

\section{B. Comparison of E/D based approach With Random Cluster Head Selection Approach}

To better analyze our energy and distance (E/D) based algorithm we have created a parallel approach which selects $\mathrm{CH}$ in random fashion. In [1] $\mathrm{CH}$ is selected based on random back off time. First node whose back off time elapses will become a $\mathrm{CH}$. We have used this concept for creating parallel approach known as random $\mathrm{CH}$ selection approach for comparing with our energy and distance (E/D) based method.

We simulate both algorithms in NS2.34 and each simulation is executed 8-10 times and comparative graphs are plotted considering the mean value. In Fig. 4 we can observe that nodes started dying earlier using random $\mathrm{CH}$ selection approach then our energy and distance (E/D) based approach. Total energy consumed by the network 
for transmitting data is more in random $\mathrm{CH}$ selection approach as compared to our method as shown in Fig. 5 Also packet collection rate is higher than the random $\mathrm{CH}$ selection approach as seen in Fig. 6.

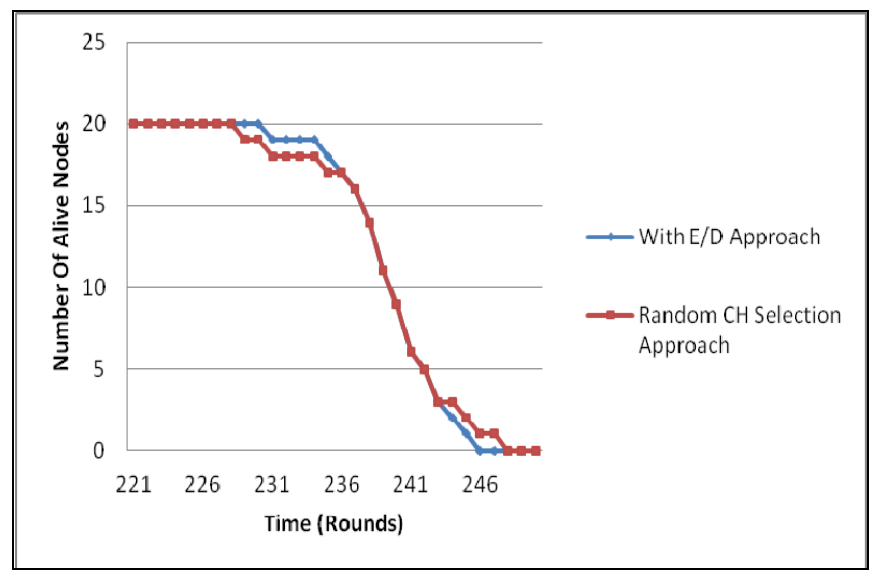

Fig.4. Comparison on Alive Nodes

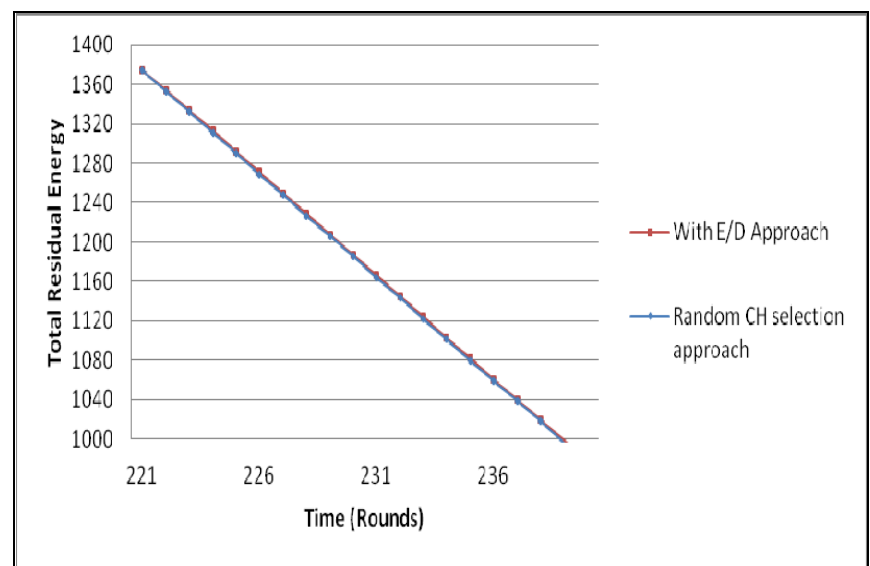

Fig.5. Comparison on Total Residual Energy

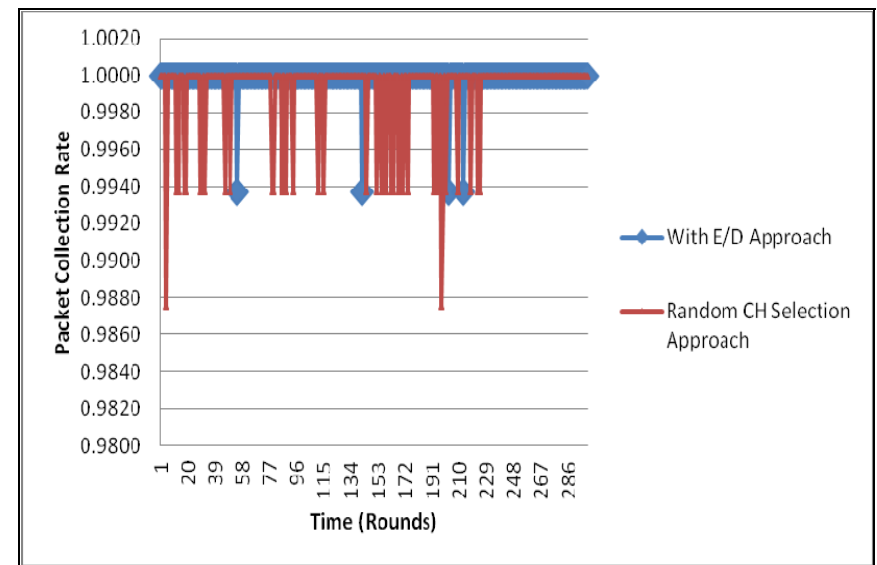

Fig. 6. Comparison on Packet Collection Rate

\section{VI.CONCLUSION}

In this paper a new energy and distance (E/D) based approach of selecting $\mathrm{CH}$ in circular clustering has been proposed. The key factors for $\mathrm{CH}$ selection are residual energy of a node and distance of a node from the sink. These two factors are very important to strengthen the life time of WSN. The experimental analysis is carried on three parameters namely number of alive nodes, total residual energy and packet collection rates. The comparative graphs clearly show that our approach out performs the random $\mathrm{CH}$ selection approach. 


\section{REFERENCES}

[1] Hai Lin, Lusheng Wang, and Ruoshan Kong, "Energy Efficient Clustering Protocol for Large Scale Sensor Networks" IEEE Sensors Journal, vol. 15, no. 12, pp.7150 - 7160, December 2015.

[2] J. N. Al-Karaki and A. E. Kamal, "Routing techniques in wireless sensor networks: A survey," IEEE Wireless Communications, vol. 11, no. 6, pp. 6-28, December 2004.

[3] O. Boyinbode, H. Le, A. Mbogho, M. Takizawa, Poliah, "A survey on clustering algorithms for wireless sensor networks," in Proceeding of 13th International Conference on Network based Information Systems (NBiS), pp. 358-364, January 2011.

[4] W. B. Heinzelman, A. P. Chandrakasan, and H. Balakrishnan, "An application-specific protocol architecture for wireless microsensor networks," IEEE Transaction on Wireless Communications, vol. 1, no. 4, pp. 660-670, October 2002.

[5] W.R Heinzelman, A.P. Chandrakasan, and B. Balakrishnan, "Energy-Efficient Communication Protocol for Wireless microsensor networks", Proceedings of 33rd annual Hawaii International Conference on System science, January 2000.

[6] Ye, Mao, et al. "EECS: an energy efficient clustering scheme in wireless sensor networks." In Performance, Computing, and Communications Conference, IPCCC, 24th IEEE International. IEEE, April 2005.

[7] O. Younis and S. Fahmy, "HEED: A hybrid, energy-efficient, distributed clustering approach for ad hoc sensor networks," IEEE Transactions on Mobile Computing, vol. 3, no. 4, pp. 366-379, October - December 2004.

[8] A. Thakkar and K. Kotecha, "Cluster head election for energy and delay constraint applications of wireless sensor network," IEEE Sensors Journal, vol. 14, no. 8, pp. 2658-2664, August 2014.

[9] Y. Liao, H. Qi, and W. Li, "Load-balanced clustering algorithm with distributed self-organization for wireless sensor networks," IEEE Sensors Journal, vol. 13, no. 5, pp. 1498-1506, May 2013

[10] M. Tarhani, Y. S. Kavian, and S. Siavoshi, "SEECH: Scalable energy efficient clustering hierarchy protocol in wireless sensor networks," IEEE Sensors Journal, vol. 14, no. 11, pp. 3944-3954, November 2014.

[11] Cheng, Lu, Depei Qian, and Weiguo Wu. "An energy efficient weight-clustering algorithm in wireless sensor networks."In Frontier of Computer Science and Technology, FCST'08. Japan-China Joint Workshop on. IEEE, December 2008.

[12] Abbasi, Ameer Ahmed, and Mohamed Younis. "A survey on clustering algorithms for wireless sensor networks." Computer communications, vol.30, no. 14, October 2007.

[13] K. Yedavalli and B. Krishnamachari, "Sequence-based localization in wireless sensor networks," IEEE Transactions on Mobile Computing, vol. 7, no. 1, pp. 81-94, January 2008.

[14] Yan, Bin, et al. "A Grid-based clustering method for large-scale wireless sensor networks." In Communications, Circuits and Systems, ICCCAS. International Conference on. IEEE, July 2007.

[15] R. Xie and X. Jia, "Transmission-efficient clustering method for wireless sensor networks using compressive sensing," IEEE Transactions on Parallel and Distributed Systems, vol. 25, no. 3, March 2014.

[16] Baek, Jinsuk, Sun Kyong An, and Paul Fisher. "Dynamic cluster header selection and conditional re-clustering for wireless sensor networks." IEEE Transactions on Consumer Electronics, vol. 56, no. 4, November2010 